\title{
Updates to the Management of Kidney Cancer
}

\author{
Presented by Eric Jonasch, MD
}

\begin{abstract}
Several updates were made to the 2018 NCCN Guidelines for Kidney Cancer. Adjuvant sunitinib is the first adjuvant therapy to be endorsed by the panel for patients with stage II and III clear cell histology renal cell carcinoma (RCC; category 2B). A promising new treatment-ipilimumab plus nivolumab for patients at intermediate and poor risk in the frontline setting-was added to the guidelines as well. Cabozantinib was added as a first-line option for poor- and intermediate-risk patients with advanced RCC.
\end{abstract}

J Natl Compr Canc Netw 2018;16(5.5):639-641 doi: 10.6004ljnccn.2018.0039

\begin{abstract}
"I $\left.\right|_{t}$ is a very exciting time in renal cell carcinoma, with new treatments. Most of which are for clear cell carcinoma," said Eric Jonasch, MD, Professor, Department of Genitourinary Medical Oncology, The University of Texas MD Anderson Cancer Center, and Vice Chair of the NCCN Guidelines Panel for Kidney Cancer, as he introduced his presentation at the NCCN 23rd Annual Conference.
\end{abstract}

\section{Adjuvant Therapy}

Five adjuvant therapy trials have been conducted in renal cell carcinoma (RCC). The ASSURE and PROTECT trials both showed negative results. ${ }^{1,2}$ ASSURE evaluated 1 year of adjuvant sunitinib versus 1 year of adjuvant sorafenib versus placebo and found no significant difference in disease-free survival (DFS) among all 3 arms, with more toxicity in the treatment arms. ${ }^{1}$ PROTECT evaluated 1 year of pazopanib versus placebo and found no significant difference in DFS between the 2 arms. $^{2}$ The SORCE ( 3 years of sorafenib vs 1 year of sorafenib vs placebo) and EVEREST (1 year of everolimus vs 1 year of placebo) trials are ongoing.

\footnotetext{
Presented by Eric Jonasch, MD, Department of Genitourinary Medical Oncology, The University of Texas MD Anderson Cancer Center, Houston, Texas.

Dr. Jonasch has disclosed that he has received consultingfees/honoraria from Bristol-Myers Squibb Company, Eisai Inc., Exelixis Inc., Novartis Pharmaceuticals Corporation, Pfizer Inc., and Roche Laboratories, Inc.; and grant/research support from Exelixis Inc., Novartis Pharmaceuticals Corporation, and Pfizer Inc.

Correspondence: Eric Jonasch, MD, Department of Genitourinary Medical Oncology, The University of Texas MD Anderson Cancer Center, 1515 Holcombe Boulevard, Unit 1374, Houston, TX 77030.

Email: ejonasch@mdanderson.org
}

More recently, the S-TRAC trial showed positive results when comparing 1 year of sunitinib versus 1 year of placebo in high-risk patients with clear cell histology. ${ }^{3}$ Adjuvant sunitinib significantly improved DFS among 615 patients by $24 \%$ in the primary analysis ( 6.8 years for sunitinib vs 5.6 years for placebo). Among higherrisk patients, median DFS was 5.9 versus 3.9 months according to investigator review. Overall survival (OS) has not been reported. ${ }^{3}$ This study led to the inclusion of adjuvant sunitinib in the updated NCCN Guidelines as a category $2 \mathrm{~B}$ recommendation for patients with stage III or higher clear cell RCC; adjuvant sunitinib was approved by the FDA in 2017.

"This recommendation may change as more data from the trial become available," Dr. Jonasch told listeners.

\section{Metastatic RCC}

The Memorial Sloan Kettering Cancer Center (MSKCC) model for risk stratification, developed by Robert Motzer, MD, and colleagues, included 5 risk factors associated with worse prognosis in RCC: Karnofsky performance status <80; low serum hemoglobin level; high corrected calcium level $(10 \mathrm{mg} / \mathrm{dL})$; high lactate dehydrogenase level (300 U/L); and no nephrectomy or $<1$ year from diagnosis to treatment. ${ }^{4}$

A newer risk stratification model for prognosis in patients on tyrosine kinase inhibitors based on International Metastatic RCC Database Consortium (IMDC) criteria separates out favorable, intermediate, and poor risk. ${ }^{5}$ The IMDC model includes 6 features, adding thrombocytosis and leukocytosis to the MSKCC model and removing elevated lactate dehydrogenase level. 
Jonasch

"Immunotherapy trials are using the IMDC criteria and they may be important for treatment selection," Dr. Jonasch said. "Therapy for advanced RCC may become increasingly tailored according to risk," he added.

Dr. Jonasch discussed recent and possible updates to the 2018 NCCN Guidelines for Kidney Cancer for treatment of advanced RCC. Dr. Jonasch noted that FDA approval was expected for ipilimumab plus nivolumab for patients at intermediate and poor risk in the frontline setting [Editor's note: this is now approved], and this treatment is now included as a category 1 recommendation, preferred indication for frontline treatment in patients with intermediate- or poor-risk features, and a category $2 \mathrm{~A}$ indication for subsequent treatment. He also anounced that cabozantinib is now approved in the frontline setting for advanced RCC. Otherwise, the guidelines remain the same as the 2017 guidelines for first- and secondline therapy.

\section{Key Questions in Metastatic Disease}

Remaining questions include:

- Can we use observation for patients with metastatic disease?

- Where do checkpoint inhibitors fit in the frontline setting?

- Is there an ideal sequence after frontline treatment failure?

- What is the role of mTOR inhibition for RCC in 2018?

Active surveillance is listed as an option in the 2018 NCCN Guidelines for favorable-risk metastatic RCC based on results of a phase II trial. ${ }^{6}$ Patients with favorable-risk - 0 or 1 IMDC factors and $\leq 2$ organs with metastatic disease-were able to delay treatment for a median of 22.2 versus 8.4 months for those in the unfavorable group $(P=.0056)$.

Cabozantinib has been added as a first-line option for relapsed or stage IV surgically unresectable advanced RCC, based on the CABOSUN study of 157 patients with intermediate or poor risk showing a significant improvement in progression-free survival (PFS) favoring cabozantinib over sunitinib (median PFS, 8.2 vs 5.6 months, respectively; Figure 1). ${ }^{\text {? }}$

CheckMate 214 evaluated the combination of nivolumab plus ipilimumab versus sunitinib as first-

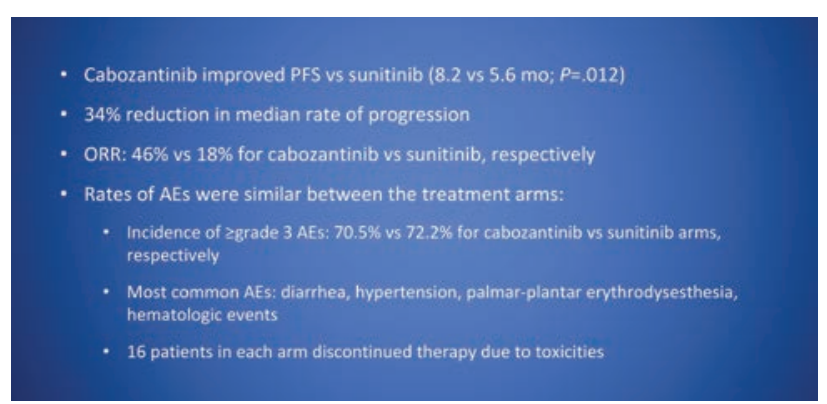

Figure 1. Efficacy and safety results of the CABOSUN study Abbreviations: AEs, adverse events; ORR, objective response rate; PFS, progression-free survival.

Data from Choueiri TK, Halabi S, Sanford BL, et al. Cabozantinib versus sunitinib as initial targeted therapy for patients with metastatic renal cell carcinoma of poor or intermediate risk: the Alliance A031203 CABOSUN trial. J Clin Oncol 2017;35:591-597.

line therapy for advanced or metastatic RCC. ${ }^{8}$ In patients with intermediate or poor risk, the immunotherapy combination achieved superior objective response rates (ORR) and duration of response (DoR) versus sunitinib. ORR was $42 \%$ versus $27 \%$, respectively $(P<.0001)$, and DoR was not reached for the immunotherapy combination versus 18.2 months for sunitinib. PFS was 11.6 versus 8.4 months, respectively, and was not significantly different. OS, however, was significantly improved with nivolumab plus ipilimumab: not yet reached versus 26 months for sunitinib $(P<.0001)$. A reversal of outcomes was seen in patients with favorable-risk cancer: median PFS was 15.3 months with the combination versus 25.1 for sunitinib $(P<.0001)$.

Patients with PD-L1-positive disease and those with PD-L1-negative disease experienced response to nivolumab plus ipilimumab, with a $16 \%$ complete response in the PD-L1-positive group and 7\% complete response rate in the PD-L1-negative group. "I don't think PD-L1 staining is necessary to decide whether to use this combination," Dr. Jonasch stated.

Treatment-related adverse events leading to discontinuation were reported in $22 \%$ of patients treated with the immunotherapy combination versus $11 \%$ of those treated with sunitinib. Immune-related adverse events led to a requirement for corticosteroids in $60 \%$ of patients, which is a downside, Dr. Jonasch noted.

The combination of atezolizumab plus bevacizumab was compared with sunitinib in 950 patients in the IMmotion 151 trial. $^{9}$ Patients were stratified according to MSKCC risk score, liver metastases, and PD-L1 status. Of these, $40 \%$ were positive for PD-L1. Median PFS was 11.2 months for the com- 
bination versus 7.7 months for sunitinib in patients with PD-L1-positive cancer, which was significant $(P=.02)$. In an intent-to-treat analysis, median PFS was 11.2 versus 8.4 months, respectively. OS was not reached in patients with PD-L1-positive disease and was 23.3 months for sunitinib. The safety of atezolizumab plus bevacizumab was improved compared with nivolumab plus ipilimumab, with only $16 \%$ of treated patients requiring steroids.

"These were the 2 big studies over the past year. We expect to have ipilimumab plus nivolumab approved," Dr. Jonasch said. He predicted that ipilimumab plus nivolumab would supersede sunitinib as first-line therapy in patients with intermediate and poor risk, but that this combination would require corticosteroids in a significant percentage of patients.

"Cabozantinib is superior to sunitinib in IMDC intermediate- and poor-risk patients, but does not achieve the complete response rates seen with ipilimumab plus nivolumab," he said.

In favorable-risk patients, sunitinib or pazopanib is preferred over ipilimumab plus nivolumab. Atezolizumab plus bevacizumab has shown promising PFS versus sunitinib in all risk categories, but thus far there is no OS signal. It is possible that in the future atezolizumab plus bevacizumab will be recommended for frontline therapy in all risk groups.

\section{Second-Line Therapy of Metastatic Disease}

Not much has changed regarding second-line therapy in the 2018 NCCN Guidelines. Subsequent therapy for patients with predominant clear cell histology includes clinical trial and cabozantinib, nivolumab, axitinib, and lenvatinib plus everolimus as category 1 recommendations. Other options include everolimus, pazopanib, sorafenib, sunitinib, and category $2 \mathrm{~B}$ recommendations for bevacizumab, high-dose interleukin-2 for selected patients, and temsirolimus. Ipilimumab plus nivolumab was just added to the NCCN Guidelines as a category 2A recommendation for subsequent therapy in patients with metastatic RCC. Best supportive care is another option.

The key question is which patients should get which drug and in what sequence, Dr. Jonasch continued. Biomarkers are needed for treatment selection. Emerging data suggest that hot tumors (with a high degree of immune infiltrate) may be associated with response to nivolumab.

"In 2018, I don't think mTOR inhibitor monotherapy is a reasonable second- or third-line option," he stated.

\section{References}

1. Haas NB, Manola J, Uzzo RG, et al. Adjuvant sunitinib or sorafenib for high-risk, non-metastatic renal-cell carcinoma (ECOG-ACRIN E2805): a double-blind, placebo-controlled, randomized, phase 3 trial. Lancet 2016;387:2008-2016.

2. Motzer RJ, Haas NB, Donskov F, et al. Randomized phase III trial of adjuvant pazopanib versus placebo after nephrectomy in patients with locally advanced renal cell carcinoma (PROTECT). J Clin Oncol 2017;35:3916-3923.

3. Ravaud A, Motzer RJ, Pandha HS, et al. Adjuvant sunitinib in high-risk renal cell carcinoma after nephrectomy. N Engl J Med 2016;375:2246-2254.

4. Motzer RJ, Mazumdar M, Bacik J, et al. Survival and prognostic stratification of 670 patients with advanced renal cell carcinoma. J Clin Oncol 1999;17:2530-2540.

5. Heng DY, Xie W, Regan MM, et al. Prognostic factors for overall survival in patients with metastatic renal cell carcinoma treated with vascular endothelial growth factor-targeted agents: results from a large, multicenter study. J Clin Oncol 2009;27:5794-5799.
6. Rini BI, Dorff TB, Elson P, et al. Active surveillance in metastatic renal-cell carcinoma: a prospective phase 2 trial. Lancet Oncol 2016:17:1317-1324.

7. Choueiri TK, Hessel C, Halabi D, et al. Progression-free survival by independent review and updated overall survival results from Alliance A031203 trial (CABOSUN): cabozantinib versus sunitinib as initial targeted therapy in patients with metastatic renal cell carcinoma [abstract]. Presented at the 2017 ESMO Congress; September 8-12, 2017; Madrid, Spain. Abstract LBA38.

8. Escudier B, Tannir N, McDermott DF, et al. CheckMate 214: efficacy and safety of nivolumab + ipilimumab v sunitinib for treatment-naïve advanced or metastatic renal cell carcinoma [abstract]. Presented at the 2017 ESMO Congress; September 8-12, 2017; Madrid, Spain. Abstract LBA5.

9. Motzer RJ, Powles T, Atkins M, et al. A phase III, open-label, randomized study of atezolizumab in combination with bevacizumab versus sunitinib in patients with untreated advanced renal cell carcinoma (IMmotion 151) [abstract]. Presented at the 2018 Genitourinary Cancers Symposium; February 8-10, 2018; San Francisco, California. Abstract 578. 